\title{
PERCEPÇÃO DE GESTORES PÚBLICOS ACERCA DA UTILIZAÇÃO DE SISTEMAS INFORMATIZADOS BASEADOS EM BLOCKCHAIN COMO FACILITADOR PARA COMPRAS SUSTENTÁVEIS
}

\author{
Victor Andrade da Silveira (Universidade Federal Fluminense) E-mail: \\ victorsilveira@id.uff.br \\ Stella Regina Reis da Costa (Universidade Federal Fluminense) E-mail: stellare@ig.com.br
}

\section{Resumo}

A preocupação com as compras sustentáveis vem ganhando grande destaque ao longo dos anos. No entanto são encontradas diversas barreiras para implementação desta prática. A falta de transparência, confiança e ética, a corrupção, centralização das informações, burocracia demasiada, dentre outros problemas, dificultam atingir objetivos sociais através das aquisições do governo. No mesmo momento em que a tecnologia blockchain avança na proposta de solucionar estas barreiras. O objetivo deste estudo foi absorver a percepção dos gestores de compras públicas sobre a utilização de sistemas de informação com funcionalidades características desta tecnologia, com intuito de reduzir estas barreiras. Este estudo é compreendido por um levantamento bibliográfico sobre a blockchain e aquisições sustentáveis, ambos aplicados ao setor público, e uma pesquisa quantitativa realizada através de questionário estruturado aplicado aos gestores das prefeituras do estado do Rio de Janeiro. Os resultados deste trabalho buscam apresentar se as barreiras encontradas podem ser minimizadas através de sistemas baseados na blockchain. Limitando-se ao contexto do setor público do estado do Rio de Janeiro.

Palavras-Chaves: compras sustentáveis, setor público, blockchain, contratos inteligentes

\section{Introdução}

A preocupação com o desenvolvimento sustentável e o impacto das empresas na sociedade e no meio ambiente torna-se evidente pelas tendências da literatura, práticas de negócios e estudos acadêmicos (WALKER E BRAMMER, 2012). Desde a Conferência de Estocolmo que a Organização das Nações Unidas (ONU) se ocupa em discutir e disseminar esta ideia, tendo seu marco principal no Relatório de Brundtland de 1987 da Comissão Mundial sobre o Meio Ambiente e o Desenvolvimento (AGU, 2016) e caminhando até os dias atuais com os 
17 objetivos para o desenvolvimento sustentável (ONU, 2020). Essas inquietações se materializaram em algumas iniciativas, sendo a Conferência das Nações Unidas sobre o Meio Ambiente e o Desenvolvimento, realizada na cidade do Rio de Janeiro em 1992, a Rio-92, a pioneira e uma das mais importantes (CABRAL E SCHRAMM, 2020).

As atividades das instituições públicas são consideradas essenciais para alavancar e manter a redução do impacto ambiental na economia, participando diretamente no equilíbrio do mercado e operando tanto como um consumidor como agente intermediário (TESTA et al., 2016). O poder de compra dos governos passa a ter grande relevância na consolidação de atividades produtivas que favoreçam o desenvolvimento sustentável (CABRAL E SCHRAMM, 2020), e são cada vez mais usados como uma ferramenta estratégica para atingir objetivos mais amplos como promover inovação, sustentabilidade, inclusão social e apoiar pequenas e médias empresas (OCDE, 2019).

Pesquisas mostram que pelas aquisições públicas é possível alcançar resultados consistentes na sociedade e que os órgãos públicos tem sido encorajados a adquirir sustentavelmente (BRAMMER E WALKER, 2011). Esta possiblidade de promover a sustentabilidade na cadeia de suprimentos motiva investigações de como a tecnologia blockchain pode solucionar barreiras aos objetivos sustentáveis (SABERI et al., 2018). Esta tecnologia é vista como uma das tendências que influenciarão os negócios e a sociedade nos próximos anos (ØLNES et al., 2017) e tem o potencial de fornecer benefícios ao governo e à sociedade além de apresentar o próximo passo no desenvolvimento do governo eletrônico, permitindo reduzir custos, compartilhar processos confiáveis e melhorar a auditoria (PALFREYMAN, 2015).

A partir deste contexto este artigo buscará identificar a percepção sobre o processo de compras e a redução das barreiras para aquisições públicas sustentáveis através da utilização de sistemas informatizados, abordando características da tecnologia blockchain.

\section{Revisão bibliográfica}

\section{1 - Bibliometria}

Buscou-se primeiramente compreender o que foi produzido sobre os temas, através de uma revisão sistemática da literatura, seguindo as etapas e recomendações sugeridos por CauchickMiguel et al.(2017): seleção das fontes, acesso as bases de dados, adoção de palavras chaves, 
uso de eventuais filtros de pesquisa, leitura do título, leitura do resumo, download dos artigos decorrentes da busca, leitura dos artigos, arquivamento e busca pelo próximo artigo.

Iniciou-se pelo acesso ao sistema da Capes, "busca por área do conhecimento" e aplicouse os filtros: áreas do conhecimento = multidisciplinar, subcategoria=todas. Posteriormente conduziu-se a busca pelos artigos relacionados às palavras chaves: "blockchain" and "public sector", "blockchain" and "government", "sustainable procurement" and "blockchain", "sustainable procurement" and "public sector". E pelos filtros: tipos de documentos=artigos e artigos in press, tipos de fonte=journals, anos=1996 a 2019. Foram retornados documentos em 13 bases de dados que totalizaram 545 documentos. Destes, 33,94\% representam a base Scopus, $18,72 \%$ a Web of Science e Compendex cada uma, somando as três $71,38 \%$.

Observou-se que $26 \%$ do resultado tratava-se de documentos repetidos, $3 \%$ conference paper, $2 \%$ livros e $12 \%$ apresentaram acesso restrito ou erro para download. $27 \%$ estão relacionados ao tema blockchain, iniciando em 2015, acentuando-se nos anos de 2017 e 2018, alcançando 54 publicações, 10 anos após a publicação de Nakamoto (2009). O maior número destes artigos esteve na IEEE Access (8 publicações), seguido do Computer law \& security review (4) e os demais com no máximo de 2 publicações. Dos autores ressaltam-se Jong Hyuk Park e Pradip Kumar Sharma, ambos da Coreia do Sul com 3 publicações. Atenta-se ao estágio inicial da pesquisa sobre a tecnologia blockchain no setor público, com potencial crescimento e o foco nas criptomoedas.

Dos $57 \%$ que representam os artigos sobre compras públicas sustentáveis, a maioria dos artigos foram publicados pelo Journal of Cleaner Production (20 publicações), seguido do Journal of Construction Engineering and Management (8) e Sustainability (5). Sobre este tema observa-se um crescimento desde 1998 e que 61\% das publicações ocorreram entre 2013 e 2014. Dentre os autores destacam-se Peter E. D. Love (7 publicações), seguido de Helen Walker (6), Lutz Preuss e Jim Smith ambos (5).

\subsection{Aquisições públicas sustentáveis}

Os contratos públicos referem-se às aquisições feitas pelas organizações governamentais ou pelo setor público, sendo uma política de múltiplos objetivos, principalmente a garantia da qualidade dos serviços e o consumo responsável (BRAMMER e WALKER, 2012). Economicamente, as aquisições públicas são reconhecidas como meio de eficiência e eficácia dos gastos públicos. Deve-se considerar também objetivos políticos mais amplos, como inovação, 
sustentabilidade, inclusão social e apoio a pequenas empresas (OCDE, 2019). O poder público é um grande fomentador do mercado e isso lhe dá o poder para exigir de seus fornecedores produtos e serviços que equilibrem as necessidades econômicas, sociais e ambientais das gerações presentes e futuras (CABRAL E SCHRAMM, 2020). Este poder de compra soma mais de 1 trilhão de Euros, 12\% do Produto Interno Bruto (PIB), na União Europeia (OCDE, 2019). No Brasil movimentam cerca de 20\% do PIB (CABRAL E SCHRAMM, 2020).

O conceito de compras sustentáveis está associado à ideia de desenvolvimento sustentável citado pelo relatório de 1987 da ONU passando pela Rio 92, e pela Cúpula Mundial sobre Desenvolvimento Sustentável em 2002 em Joanesburgo (ICLEI, 2015). Podendo ser compreendido como o processo através do qual as autoridades públicas procuram adquirir bens e serviços com impacto ambiental reduzido ao longo do ciclo de vida (TESTA et al., 2016). Visando atender objetivos de desenvolvimento por meio do processo de compra, incorporando aspectos sociais, ambientais e econômicos (BRAMMER e WALKER, 2012). Significa então alcançar uma boa relação custo/benefício com base no ciclo de vida do produto gerando benefícios não apenas para a organização, mas também para a sociedade, meio ambiente e economia (LUTTENBERGER E RUNKO LUTTENBERGER, 2016). Gerando o bem-estar social e a efetivação dos direitos sociais, dentre outros (AGU, 2016), ilustrado na figura 1.

Figura 1: Objetivos compras sustentáveis

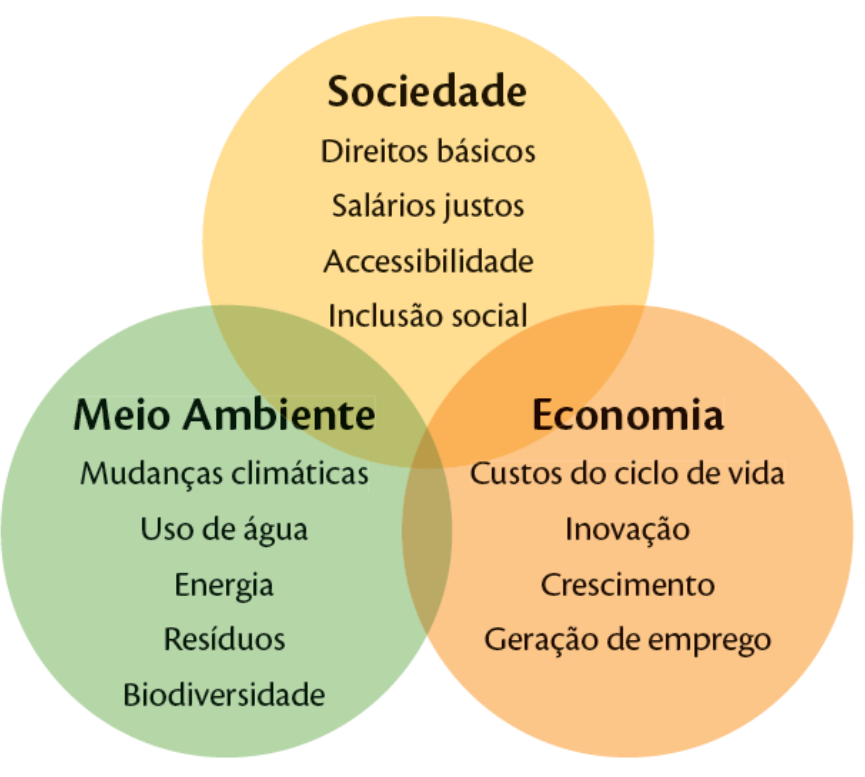

Fonte: ICLEI (2015). 
Cabe ainda ressaltar a preocupação com as questões éticas da cadeia de suprimentos, que da responsabilidade social corporativa e da ética nos negócios (WALKER E PHILLIPS, 2009).

\section{3 - A tecnologia blockchain}

Uma ou mais pessoas conhecida(s) como Satoshi Nakamoto, delineou(ram) um novo protocolo para um sistema ponto a ponto usando criptomoeda chamada Bitcoin (ABELSETH, 2018), denominado blockchain. Originado em 2009 quando Nakamoto descreveu a criptomoeda como um modelo de transações par-a-par (WEISS E CORSI, 2017). Neste modelo a cada novo registro, cópias dos dados são criadas (SABERI et al., 2018), solucionando um problema fundamental de transações na internet, a confiança (ALBELSETH, 2018). Para Paech (2017) a maneira mais fácil de entender a blockchain é pensar nela como um banco de dados na internet para armazenar valor, onde todos os participantes da rede mantêm uma cópia idêntica. Objetiva criar um ambiente descentralizado onde nenhum intermediário esteja no controle das transações e dos dados (YLI-HUUMO et al., 2016).

Este livro-razão distribuído armazena dados de transação nos “nós” (WEISS E CORSI, 2017), que são computadores interligados ao redor do mundo (TAPSCOT E TAPSCOT, 2016). Esta descentralização é uma propriedade importante, pois as mesmas informações estão em nós diferentes e só são confirmadas quando houver um consenso sobre a informação entre os nós (KOUHIZADEH E SARKIS, 2018). Novas transações são adicionadas, mas informações anteriores não podem ser removidas (ØLNES et al., 2017), assim mantém-se uma lista crescente garantindo a integridade do sistema, mesmo em face de desonestidade (SABERI $e t$ al., 2018).

Sua estrutura é encadeada, composta por um cabeçalho, incluindo um hash (cabeçalho criptografado), contendo seu próprio valor, os dados do bloco e o hash do bloco ligado a ele. A criação de um novo bloco é conhecida como "mineiração" (ØLNES et al., 2017). Uma vez que o esforço tenha sido gasto para satisfazer uma prova de trabalho, o bloco não pode ser alterado (NAKAMOTO, 2009). Todos os nós são constantemente atualizados com as informações das transações mais recentes, consequentemente todas as informações estão disponíveis em qualquer lugar, em qualquer nó, em qualquer tempo (PAECH, 2017). Existem dois tipos de cadeias de blocos: público e privado. Bitcoin é um exemplo de blockchain público, aberta a qualquer pessoa, a cadeia privada o acesso à rede é restrito porque nem todos podem participar (JOHNSON, 2017) 
A blockchain viabilizou a criação de contratos inteligentes, programas de computador que residem nesta tecnologia, executados automaticamente (PRADANA, 2018). Estes contratos dão origem ao conceito de "organizações autônomas descentralizadas" (DAOs), a forma mais complexa de um contrato inteligente (LYRA, 2019). Operam de forma autônoma sem intervenção humana, com base nas regras programadas e capazes até mesmo de firmar novos contratos (PAECH, 2017). Viabilizando aspectos de governança corporativa tradicional usando software, possibilitando os benefícios das estruturas corporativas formais e ao mesmo tempo flexibilizando a escala dos grupos informais on-line (LYRA, 2019).

\section{Metodologia}

Este trabalho iniciou com uma revisão sistemática da literatura, com intuito de proporcionar maior familiaridade com o problema, com vistas a torná-lo mais explícito (GIL,2010). Posteriormente realizou-se a aplicação de um questionário estruturado e a análise os dados através de estatística descritiva. As características e as ferramentas estatísticas dos bons estudos quantitativos são recursos preciosos (GRAY, 2012).

As unidades de análise foram os setores de compras das 92 prefeituras do estado do Rio de Janeiro, das quais 2 não foi possível o acesso, resultando então em uma população amostral de 90 gerentes de compras e licitações. Os dados foram obtidos por fontes primárias, através do envio do questionário com 17 perguntas. Segundo Gray (2012) os questionários, adequados aos objetivos da pesquisa, possibilitam perguntas padronizadas e uma abordagem analítica explorando as relações entre variáveis. Os enunciados basearam-se na literatura, buscando compreender as barreiras para utilização de compras públicas sustentáveis. Após a obtenção dos e-mails dos departamentos responsáveis, ocorreram os envios de setembro de 2019 a janeiro de 2020.

\section{Análise e discussão da pesquisa}

Dos questionários enviados obteve-se uma taxa de retorno de $24 \%$, considerada aceitável por Hair et. al (2006). As respostas foram tabuladas em planilha eletrônica, permitindo a análise da escala Likert composta de 5 itens com pesos de 1 a 5.

Ao examinar a falta de conhecimento sobre como especificar critérios sustentáveis nos termos de referência e editais, encontrou-se que 90\% dos respondentes escolheram as opções concordo ou concordo totalmente de que há dificuldade. Este resultado expõe que a 
desinformação é uma barreira às aquisições sustentáveis. Zaidi et. al. (2018) identificaram como um grande problema a falta de informação sobre os produtos com influências sobre a cadeia de suprimentos. Para McMurray et. al. (2014) este baixo nível de compreensão ocorre entre vários níveis de funcionários, desde gerentes de compras aos gerentes de alto nível. As respostas transparecem a concepção de que há uma carência de informações sobre especificações técnicas que levem a uma aquisição mais sustentável. As respostas sobre a dificuldade em se encontrar estas especificações totalizou uma proporção de $82 \%$ pela seleção de concordo e concordo totalmente também pela falta de informações da composição de materiais e produtos sustentáveis. Complementando esta percepção Testa et. al. (2016) aponta que intensificar a informação e aumentar a conscientização sobre contratos públicos sustentáveis pode apoiar fortemente as aquisições sustentáveis. O conhecimento destas práticas pode incluir o detalhamento de requisitos, responsabilidades e experiências já realizadas por outros países (Zhu et. al., 2013).

A segunda pergunta com maior proporção de concordância foi se há dificuldade em se obter informações sobre o processo de fabricação e fornecimentos dos produtos. Para Kouhizadeh e Sarkis (2018) as informações necessárias para seleção de fornecedores e fabricantes é vista pela indústria e pelo meio acadêmico como uma questão crítica para o sucesso a longo prazo. A avaliação de fornecedores nas etapas iniciais é necessária para garantir a sustentabilidade destas cadeias. Das respostas, exceto 2 nulas, todas as demais optaram por concordar que a dificuldade em se conseguir informações sobre a cadeia de suprimentos é um obstáculo para comprovação de práticas sustentáveis, alcançando $91 \%$ da frequência relativa das opções de aprovação.

Zhu et. al. (2013) apontam como direção de futuras pesquisas a investigação de sistemas de difusão de práticas de compras públicas sustentáveis. Atendendo a esta sugestão buscou-se compreender a percepção dos gestores sobre a utilização de um sistema único entre prefeitura, fornecedores e fabricantes e se esta ferramenta seria útil na verificação das especificações e critérios sustentáveis exigidos na licitação. As respostas a este enunciado alcançaram a maior proporção de concordância (63\% - concordo totalmente e 32\% - concordo) dentre todas as questões apresentadas, totalizando 95\% das opções de aprovação. Segundo Zaidi et. al. (2018) as organizações devem projetar ferramentas que possam auxiliar neste tipo uma aquisição. É preciso que haja a oferta de ferramentas que promovam o conhecimento necessário para implementar efetivamente as práticas de sustentáveis (ZHU et. al., 2013). Para Kouhizadeh e Sarkis (2018) a barreira de desinformações pode ser efetivamente aliviada usando a tecnologia blockchain. Dados históricos de desempenho e sustentabilidade dos fornecedores podem ser 
disponibilizados, facilitando aos funcionários examinarem práticas e registro internos de economia de energia e redução de poluição para determinar a proatividade relativa das práticas ambientais dos fornecedores (ZHU et. al., 2013).

Almejou-se ainda assimilar a ideia dos gestores sobre esta complexidade e especificidade da composição dos diversos produtos e de que forma se tornam uma barreira para transcrevê-los nos documentos da licitação. Apenas 5\% discordaram que não há dificuldade e $68 \%$ que concordaram que é complexo. Uma solução seria a utilização de um sistema que integrasse toda a cadeia. As informações compartilhadas na blockchain oferecem às organizações a oportunidade de acompanhar e selecionar seus fornecedores em diferentes níveis da cadeia de suprimentos (KOUHIZADEH e SARKIS, 2018), auxiliando na catalogação dos produtos e fornecedores o que seria um avanço para este modelo e compra (GONZÁLES-BENITO et. al., 2016). Os autores concluem que a formação de um painel de fornecedores sustentáveis pode ter efeitos positivos à população na reputação dos fornecedores.

Dispositivos de Internet das Coisas (IoT) podem estar interligados a uma plataforma blockchain para coletar informações legítimas sobre os produtos e processos aumentando a confiança (PRADANA, 2018; SABERI et. al., 2017). Sarkis et. al. (2020) citam a necessidade de acelerar ações sustentáveis após a pandemia do COVID-19 e a implementação de sistemas de logística mais inteligentes e logística reversa para materiais e produtos ativados pelas tecnologias IoT. Demais estudos transmitem que há a possibilidade de os sistemas de informação, consorciados às tecnologias da quarta revolução industrial (Indústria 4.0)( PRADANA, 2018).

Foi possível também identificar que 55\% dos gestores apontaram que não realizam a análise do ciclo de vida para se tomar a decisão da compra. Testa et. al. (2016), cita que a conscientização de compras públicas pode significar a mudança de uma estratégia focada apenas no custo para a orientação ao custo do ciclo de vida. Práticas de compras públicas sustentáveis precisam considerar então a produção, o uso e o descarte dos produtos, no entanto essa perspectiva nem sempre é fácil de ser avaliada (ZHU et. al., 2013). No contexto de compras públicas sustentáveis é essencial para demonstrar que os processos e decisões de compras precisam ir além, pois o preço de compra não reflete os ganhos financeiros e não financeiros oferecidos por ativos ambiental e socialmente melhores (TESTA et. al., 2016). A possibilidade de se rastrear um produto desde sua produção até o descarte pelo blockchain, permite também, através deste histórico, facilitar o cálculo do valor 
de todo o ciclo de vida do produto, e criar uma base com estimativas cada vez mais seguras (LUTTENBERGER E RUNKO LUTTENBERGER, 2016).

Com $77 \%$ de concordância, os gestores apontaram que a influência de funcionários antiéticos e mal intencionados sobre as aquisições sustentáveis, sendo estes responsáveis pela elaboração dos editais, com uma barreira. Os gestores transmitiram a percepção de que há uma influência negativa destas pessoas mal intencionadas sobre a especificação dos produtos e serviços. Pradana et. al. (2018) apontam que uma revolução tem ocorrido pela tecnologia blockchain em processos sem confiança através da substituição de intermediários antiéticos por contratos inteligentes, incluindo agentes corruptos. Funcionários e intermediários mal intencionados aproveitam que os processos de compras são difíceis de serem auditados por órgãos de controle ou por cidadão interessados (MCMURRAY et. al., 2014). Entretanto os benefícios básicos do blockchain estão relacionados à melhoria da integridade dos dados e às transações que são irrefutáveis, o que, por sua vez pode resultar na rastreabilidade das informações e transparência, que apoiam a redução da corrupção e a fraude (ØLNES et. al., 2017).

Pela compreensão dos respondentes $73 \%$ concordaram em ser um modelo de sistemas útil o conceito de DAOs. Após a escolha dos produtos e materiais necessários à administração, um DAO poderia assumir a operacionalização das compras, já com um painel de fornecedores e produtos, com preços baseados no custo do ciclo de vida. O processo de compras pode ser automatizado, integrando as etapas de pregões eletrônicos, eliminando a favorecimento de fornecedores e preços. Com esta tecnologia fraudes podem ser evitadas devido à fidelidade e transparência, sendo possível ainda afastar agentes antiéticos e responsabilizar os corruptos por danos sociais (SABERI et al., 2018).

Quanto à falta de transparência, com $82 \%$ das respostas foram para concordo e concordo totalmente de que esta pode ser uma barreira para as aquisições sustentáveis. Pradana et. al., (2018) cita que a blockchain fornecerá transparência ao sistema já que todas as partes envolvidas tem acesso às informações. Para Saberi et. al. (2018) o maior impacto que a blockchain pode causar na cadeia de suprimentos é a sua capacidade de melhorar a colaboração entre os interessados e assim melhorar sua eficiência, transparência e agilidade.

\section{Considerações finais}

Considerando o papel relevante das aquisições públicas para a sustentabilidade, a utilização de uma ferramenta com as características da blockchain apresenta-se com grande potencial 
(KOUHIZADEH e SARKIS, 2018; SABERI et. al., 2018). O blockchain pode ajudar a sustentabilidade através da garantia dos direitos humanos e oferecer melhor garantia dos direitos trabalhistas a partir da possibilidade de rastreabilidade do produto alcançando seus fornecedores e fabricantes (SABERI et al., 2018). O rastreio dos produtos e produtores permitiria a fiscalização pelos órgãos públicos da utilização de mão-de-obra infantil e escrava, cumprimento de leis trabalhistas e demais atividades relacionadas ao processo de fabricação. Possibilitaria a criação de uma lista de fornecedores (GONZÁLES-BENITO et. al., 2016), integrado a um catálogo de produtos sustentáveis considerando seu ciclo de vida (LUTTENBERGER E RUNKO LUTTENBERGER, 2016; ZHU et. al., 2013). Um sistema descentralizado possibilitaria compras consorciadas entre prefeituras, visando a redução do custo e assegurando os princípios da aquisição pública e a eficiência, transparência, confiança, auditoria, integridade e evitando a corrupção (ØLNES et. al., 2017).

\section{REFERÊNCIAS}

AGU - ADVOCACIA-GERAL DA UNIÃO. Carvalho, Maria G. Ferreira, Maria Augusta Soares de O. Villac, Teresa. Guia Nacional de Licitações Sustentáveis. (2016).

BRAMMER, Stephen and WALKER, Helen. Sustainable procurement in the public sector: an international comparative study. International Journal of Operations \& Production Management. 2011, Vol. 31 pp. $452-476$.

CAUCHICK-MIGUEL, Paulo A; Campos, LUCILA M. de Souza; JABBOUR, Charbel J. Chiappetta e JABBOUR, Ana Beatriz L. de Sousa. Elaboração de Artigos Acadêmicos: Estrutura Métodos e Técnicas. Rio de Janeiro: Elsevier (2017).

GRAY, David E. Pesquisa no Mundo Real. Porto Alegre: Penso (2012).

GIL, Antonio Carlos. Como Elaborar Projeto de Pesquisa. São Paulo. Atlas, 2010.

GONZÁLEZ-BENITO, Javier; LANNELONGUE, Gustavo; FERREIRA, Luis Miguel and GONZALEZ-ZAPATERO, Carmen. The effect of green purchasing on purchasing performance: the moderating role played by long-term relationships and strategic integration. Journal of Business \& Industrial Marketing. 2016, p. 312-324

HAIR, J.F.; ANDERSON, R.E.; TATHAM, R.L. and BLACK, W.C. Multivariate Data Analysis, Fifth ed. Prentice-Hall, Englewood Cliffs, 1998, 677-644.

ICLEI - Governos Locais pela Sustentabilidade. Manual Procura+ Um Guia para Implementação de Compras Públicas Sustentáveis. $3^{\mathrm{a}}$ ed. São Paulo, 2015.

JOHNSON, Abby. An Introduction to Blockchain. Darden Business Publiching. 2017.

KOUHIZADEH, Mahtab and SARKIS, Joseph. Blockchain Practices, Potentials, and Perspectives in Greening Supply Chains. Sustainability. 2018, 3652, 10;

LUTTENBERGER, Axel and RUNKO LUTTENBERGER, Lidija. Sustainable procurement and environmental life-cycle costing in maritime transport. WMU Journal of Maritime Affairs. 2017, 16:219-231 LYRA, João Guilherme. Blockchain e Organizações Descentralizadas. Brasport. 2019. 
MCMURRAY, Adela; ISLAM, Md; CHAMHURI, Siwar and FIEN, John. Sustainable procurement in Malaysian organizations: Practices, barriers and opportunities. Journal of Purchasing and Supply Management. 2014, 20, 195-207.

CABRA, Luciana Priscila Barros and SCHRAMM, Vanessa Batista. Guia de Critérios mensuráveis para compras públicas sustentáveis no Brasil, 2020. Disponível em: < http://dspace.sti.ufcg.edu.br:8080/jspui/bitstream/riufcg/12750/1/LUCIANA\%20PRISCILA\%20BARROS\%20C ABRAL\%20-\%20DISSERTA\%c3\%87\%c3\%830\%20\%28PPGA\%29\%202020.pdf> Acesso em: 26 maio 2020.

NAKAMOTO, Satoshi. Bitcoin: A Peer-to-Peer Electronic Cash System, 2009. Disponível em: < www.bitcoin.org>. Acessado em: 20 jul. 2019.

OCDE, Organisation for Economic Cooperation and Development. Government at a Glance 2019, 2019. Disponível em: <https://www.oecd-ilibrary.org/governance/government-at-a-glance-2019_8ccf5c38-en> Acesso em: 08 mar. 2020.

ØLNES, Svein. UBACHT, Jolien. JANSSEN, Marijn. Blockchain in government: Benefits and implications of distributed ledger technology for information sharing. Government Information Quarterly. 2017.

ONU, Organização das Nações Unidas. About the Sustainable Development Goals, 2020. Disponível em: <https://www.un.org/sustainabledevelopment/sustainable-development-goals/> Acesso em: 08 mar. 2020

PAECH, Philipp. The Governance of Blockchain Financial Networks. The Modern Law Review. (2017)

PRADANA, Aditya; SING, Goh Ong; KUMAR, Yogan Jaya and MOHAMMED, Ali A. Blockchain Traffic Offence Demerit Points Smart Contracts: Proof of Work. International Journal of Advanced Computer Science and Applications. 2018, Vol. 9, No. 11.

SABERI, Sara; KOUHIZADEH, Mahtab; SARKIS, Joseph and SHEN, Lejia. Blockchain technology and its relationships to sustainable supply chain management. International Journal of Production Research. 2018, 57:7, 2117-2135.

SARKIS, Joseph; COHEN, Maurie J. and DEWICK, Paul. A brave new world: Lessons from the COVID-19 pandemic for transitioning to sustainable supply and production. Resources, Conservation and Recycling. 2020, Vol. 159.

TAPSCOTT, Don. TAPSCOTT, Alex. Blockchain Revolution: Como a tecnologia por trás do Bitcoin está mudando o dinheiro, os negócios e o mundo. São Paulo: SENAI-SP (2016).

TESTA, Francesco. ANNUNZIATA, Eleonora. IRALDO, Fabio. FREY, Marco. Drawbacks and opportunities of green public procurement: an effective tool for sustainable production. Journal of Cleaner Production. (2016)

YLI-HUUMO, Jesse; KO, Deokyoon; CHOI, Sujin; PARK, Sooyong and SMOLANDER, Kari. Where Is Current Research on Blockchain Technology?-A Systematic Review. PLOS ONE. 2016, 11(10).

WALKER, Helen. BRAMMER, Stephen. The relationship between sustainable procurement and eprocurement in the public sector. Int. J. Production Economics. (2012).

WALKER, Helen. PHILLIPS, Wendy. Sustainable procurement: emerging issues. Int. J. Procurement Management. (2009).

WEISS, Mitchell and CORSI, Elena. Bitfury: Blockchain for Government. Harvard Business School Case. 2017, 818-031.

ZAIDI, Syed Anees Haider, MIRZA, Faisal Mehmood; HOW, Fujun and ASHRAF, Rana Umair. Addressing the sustainable development through sustainable procurement: What factors resist the implementation of sustainable procurement in Pakistan? Socio-Economic Planning Sciences. in press. 
ZHU, Qinghua; GENG, Yong and SARKIS, Joseph. Motivating green public procurement in China: An individual level perspective. Journal of Environmental Management. 2013, p. 85-95. 